\title{
Toll-like receptor-2 and -4 are associated with hyperlipidemia
}

\author{
YA-JUN ZHU ${ }^{1}$, CHAO WANG $^{2}$, GUANGYAO SONG $^{3}$, SHA-SHA ZANG $^{1}$, YI-XUAN LIU ${ }^{1}$ and LING LI ${ }^{1}$ \\ ${ }^{1}$ Postgraduate Department, Hebei Medical University, Shijiazhuang, Hebei 050017; \\ ${ }^{2}$ Department of Clinical Medical Research Center, Hebei General Hospital, Shijiazhuang, Hebei 050051; \\ ${ }^{3}$ Department of Internal Medicine, Hebei Medical University, Shijiazhuang, Hebei 050017, P.R. China
}

Received December 2, 2014; Accepted September 22, 2015

DOI: $10.3892 / \mathrm{mmr} .2015 .4465$

\begin{abstract}
Recent studies have suggested that toll-like receptors (TLRs) contribute to insulin resistance, and that fatty acids have a role in TLR activation. Other studies have found that TLR2 and TLR4 upregulation is consistent with an increase in serum lipid. Therefore, it was hypothesized that TLRs are associated with hyperlipidemia. The aim of the present study was to investigate whether TLR 2 or TLR4 was associated with hyperlipidemia and to provide novel targets for hyperlipidemia therapy. Volunteers were selected at the Medical Examination Center of Hebei General Hospital (Shijiazhuang, China), including 43 patients with high triglyceride (TG) levels, 84 with high total cholesterol (TC) levels and 55 with high TG and high TC levels. In addition, 68 healthy volunteers were selected as a control group. For the animal study, the TLR gene and protein levels were assessed in the skeletal muscle of rats fed a high-fat diet. As expected, TLR 2 and TLR4 gene expression were upregulated when TC increased, TG increased, or TC and TG increased. In rats fed a high-fat diet, the levels of gene and protein expression in the skeletal muscle of the two TLRs were all increased compared with the control group, this was consistent with an increase in TC and TG. In addition, in drug treatment groups the mRNA and protein expression levels of TLR in the skeletal muscle of rats fed a high fat diet were decreased, as were the TC and TG levels. In conclusion,
\end{abstract}

Correspondence to: Professor Guangyao Song, Department of Internal Medicine, Hebei Medical University, 361 Zhongshan East Road, Shijiazhuang, Hebei 050017, P.R. China

E-mail: sguangyao2@163.com

Abbreviations: TLRs, Toll-like receptors; NAFLD, nonalcoholic fatty liver disease; PUFA, polyunsaturated fatty acids; TG, triglyceride; TC, total cholesterol; HTC group, high TC group; HTG group, high TG group; MHL group, mixed hyperlipidemia group; PBMCs, peripheral blood mononuclear cells; Con group, control group; NC group, negative control group; HF group, high-fat diet group; HFD group, high-fat diet control group; JLD group, high-fat diet plus Jin Li Da group; Ptz group, high-fat diet plus pioglitazone group

Key words: TLR2, TLR4, high-fat diet, hypertriglyceridemia, hypercholesterolemia, mixed hyperlipidemia these findings suggest that TLR2 and TLR4 are associated with hyperlipidemia.

\section{Introduction}

At present, hyperlipidemia is an important global health concern due to its association with obesity, insulin resistance and nonalcoholic fatty liver disease (NAFLD), which are important features of metabolic syndrome $(1,2)$. Obesity has become a global health problem, with recent statistics indicating that rates of obesity have risen among adolescents from $5 \%$ in 1970 to $>18 \%$ in 2008 , and the number of overweight adolescents now exceeds $34 \%$ in the USA $(3,4)$.

Chronic inflammation in association with insulin resistance is a key characteristic of obesity. Previous studies have found associations between chronic inflammation, hyperlipidemia and insulin resistance (5-7). A recent study found that toll-like receptors (TLRs), a family of pattern recognition receptors that contribute to congenital immunity, have an important role in the outcome and development of metabolic syndrome (8). Previous studies by Lee et al $(9,10)$ have found that saturated fatty acids activate TLR4, and that polyunsaturated fatty acids (PUFA) inhibit saturated fatty acid- and LPS-induced activation of TLR4 $(9,10)$. In addition, the saturated fatty acid lauric acid potentiates, while the $n$-3 PUFA docosahexaenoic acid inhibits lipopeptide (TLR2 agonist)-induced TLR2 activation (11). Fatty acids are able to induce or inhibit the activation of TLR2 and TLR4; however, it remains to be elucidated whether TLR 2 or TLR4 are associated with hyperlipidemia. Therefore, the present study detected TLR2 and TLR4 gene expression in peripheral blood mononuclear cells (PBMCs) among different individuals and in the skeletal muscle of rats fed a high-fat diet in order to examine the association with hyperlipidemia.

\section{Patients and methods}

Patients. Patients with high triglyceride (HTG) levels, or high cholesterol (HTC) levels aged between 25 and 55-years-old were recruited from the Medical Examination Center of Hebei General Hospital (Shijiazhuang, China). They were divided into the HTG group $(n=43)$, the HTC group $(n=84)$ or the mixed hyperlipidemia group (MHL group, $n=55$ ). In addition, 68 healthy volunteers were recruited and assigned to the control group (Con group). Participants were excluded if they 
Table I. Reverse transcription-polymerase chain reaction primer sequences.

\begin{tabular}{lll}
\hline Gene & \multicolumn{1}{c}{ Forward primer (5'-3') } & \multicolumn{1}{c}{ Reverse primer (5'-3') } \\
\hline TLR2-h & GACTTCTCCCATTTCCGTCT & GGTGTTCATTATCTTCCGCA \\
TLR4-h & ATCATTGGTGTGTCGGTCC & GCTCATTCCTTACCCAGTCC \\
GAPDH-h & TGAACGGGAAGCTCACTG & GCTTCACCACCTTCTTGATG \\
TLR2-r & TCGGGACTCACAGCAAACA & TTCACACAGGCTCGCAAGT \\
TLR4-r & TGGTCAGTGTGCTTGTGGTA & GTTTCTCACCCAGTCCTCATT \\
GAPDH-r & TGAACGGGAAGCTCACTG & GCTTCACCACCTTCTTGATG
\end{tabular}

TLR, Toll-like receptor. GAPDH, glyceraldehyde 3-phosphate degydrogenase; h, human; r, rat.

had a history of smoking, alcohol abuse or use of oral drugs, or evidence of infection within the past month (ie., C-reactive protein concentrations $>5 \mathrm{mg} / \mathrm{dl}$ ). Participants were also excluded if they had a history of any the following diseases: Diabetes mellitus, hypertension, blood disorders, heart disease, liver and renal disease or dystrophia; had undergone long-term high intensity exercise; or were pregnant or lactating $(12,13)$. HTG and HTC were defined as TG $\geq 1.7 \mathrm{mmol} / \mathrm{l}$ and TC $\geq 5.7 \mathrm{mmol} / 1$, respectively. All patients were matched for age, body mass index and waist circumference (Table II). The present study was approved by the ethics committee of the Hebei General Hospital (Hebei, China). Written informed consent was obtained from each participant.

Animals and animal care. A total of 36 male Sprague-Dawley rats (120-150 g; two week-old) were obtained from the China Experimental Animal Resources Research Institute for Food and Drug Control [license no. scxk (Beijing) 2009-0017; certificate no. 0270141; Beijing, China] and received an animal experimental licence from the same institution. All rats were housed in $12 \mathrm{~h}$ light/dark cycle conditions for 2 weeks. Initially, they were randomly divided into two groups: Negative control group (NC group; n=12) and high-fat diet group (HF group; $\mathrm{n}=24)$. Six rats were selected randomly from each group after 6 weeks to harvest blood and skeletal tissue samples for experimentation. They were anesthetized with 3\% pentobarbital sodium (30 mg/kg; Beijing Pu Bosi Biotechnology Co., Ltd., Beijing, China) and sacrificed following blood sample collection. Subsequently, the remaining rats in the HF group were randomly divided into three groups: High-fat diet control group (HFD, N=6), high-fat diet plus Jin Li Da group (JLD, n=6; Shijiazhuang Yiling pharmaceutical Co., Ltd., Shijiazhuang, China) and high-fat diet plus pioglitazone group (Ptz, n=6; Takeda Pharmaceuticals Co., Ltd., Tianjin, China). All rats were housed in a $12 \mathrm{~h}$ light-dark cycle and provided ad libitum access to a rodent standard diet $(65.5 \%$ carbohydrate, $10.3 \%$ fat and $24.2 \%$ protein) or a HFD $(20.1 \%$ carbohydrate, $59.8 \%$ fat and $20.1 \%$ protein). The drug treatments were intragastrically administered for 8 weeks (JLD $1.5 \mathrm{~g} / \mathrm{kg}$ per day and Ptz $4.5 \mathrm{mg} / \mathrm{kg}$ per day).

Isolation of PBMCs. Mononuclear cells were isolated from heparinized blood obtained from all patients who had fasted for $8 \mathrm{~h}$, by Ficoll Hypaque centrifugation followed by magnetic separation using the depletion technique (Miltenyi Biotec,
Auburn, CA, USA) as described previously (14-16). Using this technique, $>92 \%$ of cells were identified as monocytes by CD14 staining. Whole blood sample was collected following medical examination PBMCs were isolated within $3 \mathrm{~h}$

TC and TG detection. All blood samples were assayed using a Hitachi 7300-110 apparatus (Hitachi, Ltd., Tokyo, Japan). The data were obtained from the Medical Examination Center of Hebei General Hospital.

TLR2 and TLR4 mRNA expression. RNA was extracted from monocytes or skeletal muscles using TRIzol reagent (Invitrogen Life Technologies, Carlsbad, CA, USA) according to the manufacturer's instructions. The RNA quality was assessed using a NanoDrop 1000 spectrophotometer (Thermo Fisher Scientific, Waltham, MA, USA). cDNA was synthesized using the iScript cDNA synthesis kit (Bio-Rad Laboratories, Inc., Hercules, CA, USA). Reverse transcription-quantitative polymerase chain reaction (RT-qPCR) was performed on an ABI PRISM 7300 PCR system (Applied Biosystems Life Technologies, Foster City, CA, USA) using SYBR Green I GoTaq ${ }^{\circledR}$ qPCR Master mix (Promega Corporation, Madison, WI, USA) with GAPDH as a control and a mixed cDNA sample control incorporated into each PCR run $(17,18)$. All target gene primers were designed with DNAMan 6.0.40 (Lynnon Biosoft, San Ramon, CA, USA) and the primers are shown in Table I. PCR was performed as follows: Denaturation at $95^{\circ} \mathrm{C}$ for $10 \mathrm{~min}$, followed by 40 cycles of $95^{\circ} \mathrm{C}$ for $15 \mathrm{sec}, 58^{\circ} \mathrm{C}$ for $20 \mathrm{sec}$ and $72^{\circ} \mathrm{C}$ for $27 \mathrm{sec}$. Subsequently, the PCR products were assessed using a melting curve analysis to confirm the specificity of the amplification. The mRNA expression of target genes was expressed as a ratio to glyceraldehyde 3-phosphate dehydrogenase (GAPDH) (12).

Western blot analysis. Frozen-dried muscle tissues $(50 \mathrm{mg})$ were homogenized in $100 \mu \mathrm{g} / \mathrm{ml}$ phenylmethylsulfonyl fluoride (Beijing Solarbio Science \& Technology Co., Ltd., Beijing, China), including three cycles of homogenization for $8 \mathrm{sec}$ and then standing for $10 \mathrm{~min}$. The samples were subjected to centrifugation at $10,000 \mathrm{x}$ g for $10 \mathrm{~min}$ at $4^{\circ} \mathrm{C}$. The protein concentration of the supernatant was determined using a NanoDrop 1000 spectrophotometer (Thermo Fisher Scientific). Muscle protein fractions $(30 \mu \mathrm{g})$ were separated by $10 \%$ SDS-polyacrylamide gel (Sigma-Aldrich, St. Louis, MO, USA) electrophoresis and transferred onto polyvinylidene 
Table II. Comparison of clinical parameters between groups (mean \pm standard deviation).

\begin{tabular}{lcccc}
\hline Parameter & Con group & HTC group & HTG group & MHL group \\
\hline Gender (male/female) & $40 / 28$ & $51 / 33$ & $27 / 16$ & $35 / 20$ \\
Age (years) & $34.68 \pm 4.27$ & $35.83 \pm 5.05$ & $35.37 \pm 4.57$ & $35.27 \pm 4.53$ \\
BMI $\left(\mathrm{kg} / \mathrm{m}^{2}\right)$ & $23.88 \pm 1.29$ & $23.40 \pm 1.68$ & $24.37 \pm 1.90$ & $24.58 \pm 2.06$ \\
Waist $(\mathrm{cm})$ & $82.97 \pm 7.52$ & $82.44 \pm 8.36$ & $82.44 \pm 6.54$ & $85.29 \pm 9.42$ \\
TC $(\mathrm{mmol} / \mathrm{l})$ & $4.46 \pm 0.63$ & $6.12 \pm 0.20^{\mathrm{a}}$ & $4.82 \pm 0.41^{\mathrm{a}}$ & $6.20 \pm 0.30^{\mathrm{a}}$ \\
TG $(\mathrm{mmol} / \mathrm{l})$ & $0.87 \pm 0.30$ & $1.18 \pm 0.22^{\mathrm{a}}$ & $2.66 \pm 0.45^{\mathrm{a}}$ & $2.64 \pm 0.49^{\mathrm{a}}$
\end{tabular}

${ }^{\mathrm{a}} \mathrm{P}<0.05$, compared with the Con group. HTC, high total cholesterol; HTG, high triglyceride; BMI, body mass index; TC, cholesterol; TG, triglyceride; MHL, mixed hyperlipidemia.

Table III. Comparison of relative TLR gene expression levels (mean \pm standard deviation).

\begin{tabular}{lcccc}
\hline Gene & Con group & HTC group & HTG group & MHL group \\
\hline TLR2 & $0.98 \pm 0.32$ & $1.45 \pm 0.54^{\mathrm{a}}$ & $1.26 \pm 0.33^{\mathrm{a}}$ & $1.34 \pm 0.42^{\mathrm{a}}$ \\
TLR4 & $1.01 \pm 0.39$ & $2.08 \pm 0.51^{\mathrm{a}}$ & $1.56 \pm 0.30^{\mathrm{a}}$ & $1.63 \pm 0.41^{\mathrm{a}}$ \\
\hline
\end{tabular}

${ }^{a} \mathrm{P}<0.05$, compared with the Con group. TLR, Toll-like receptor; HTC, high cholesterol; HTG, high triglyceride; MHL, mixed hyperlipidemia.

difluoride (PVDF) membranes (EMD Millipore, Billerica, MA, USA). Following protein transfer, the membranes were blocked with $5 \%$ non-fat dry milk in Tris-buffered saline containing $0.05 \%$ Tween-20 (TBST) overnight at $4^{\circ} \mathrm{C}$. Following blocking, the membranes were incubated overnight at $4^{\circ} \mathrm{C}$ with anti-TLR2 (polyclonal rabbit anti-mouse; cat. no. bs-1019R; 1:300, BIOSS, Beijing, China), anti-TLR4 (polyclonal rabbit anti-mouse; cat. no. BA1717; 1:200, Wuhan Boster Biological Technology, Ltd., Wuhan, China) and anti- $\beta$-actin antibodies (polyclonal rabbit anti-mouse; cat. no. 85-14-6496-82; 1:5,000, eBioscience, Inc., San Diego, CA, USA). They were then incubated with the appropriate horseradish peroxidase-conjugated secondary antibodies (polyclonal goat anti-rabbit; cat. no. SA00002-2; 1:10,000, ProteinTech Group, Inc., Chicago, IL, USA) for $1 \mathrm{~h}$ at room temperature. All antibodies were diluted with TBST. The membranes were washed three times for $10 \mathrm{~min}$ in TBST. The immunoreactive proteins were visualized by enhanced chemiluminescence (Pierce Biotechnology, Rockford, IL, USA). X-ray film (Ruike Medical Devices Co., Ltd., Xiamen, China) was exposed to the PVDF membranes for $5 \mathrm{~min}$. The reaction product of each blot was analyzed by densitometry using Bandscan 5.0 software (http://soft.bio1000. com/show-149.html).

Statistical analysis. All data analysis was performed with SAS 8.0 software for Windows XP (Hebei Medial University, Shijiazhuang, China). The key outcome variables were compared between study groups and the Con group using unpaired two-sample/group t-tests for continuous variables, and $\chi^{2}$ or Fisher's exact test, were used for categorical variables. $\mathrm{P}<0.05$ was considered to indicate a statistically significant difference.

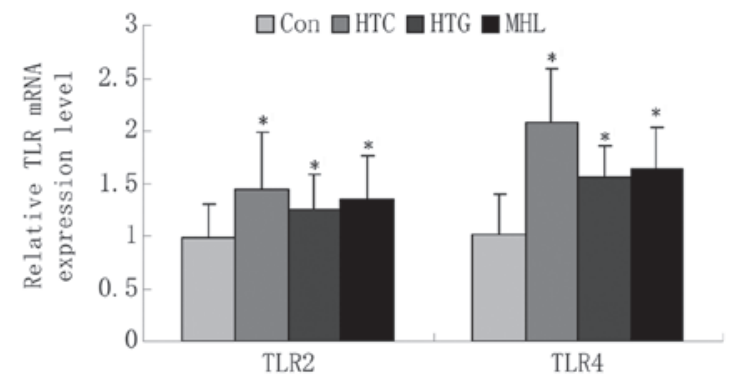

Figure 1. Comparison of TLR2 and TLR4 gene expression in peripheral blood mononuclear cells, as determined by reverse transcription-quantitative polymerase chain reaction. ${ }^{*} \mathrm{P}<0.05$. TLR, Toll-like receptor; HTC, high cholesterol; HTG, high triglyceride; Con, control; MHL, mixed hyperlipidemia.



Figure 2. Comparison of TG and TC levels between the Con and HF diet groups as determined by the $7300-110$ apparatus. ${ }^{*} \mathrm{P}<0.05$, compared with the Con group. TC, total cholesterol; TG, triglyceride; HF, high-fat diet group; Con, control.

\section{Results}

TLR2 and TLR4 are highly expressed in patients with hyperlipidemia. The gene expression of the TLRs are shown in Table III 


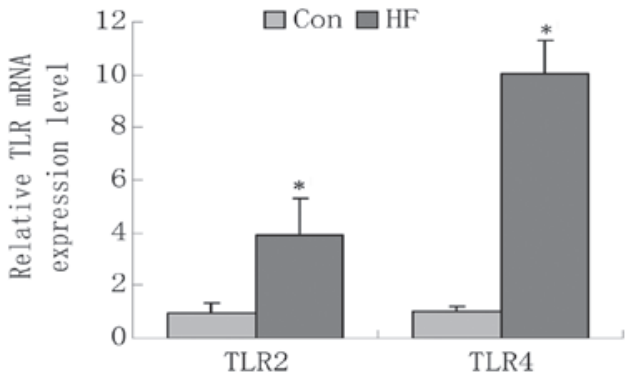

Figure 3. Comparison of TLR gene expression in the skeletal muscle of the control group and the high-fat diet group as determined by reverse transcription-quantitative polymerase chain reaction. ${ }^{*} \mathrm{P}<0.05$, compared with the Con group. HF, high-fat diet group; TLR, Toll-like receptor; Con, control.

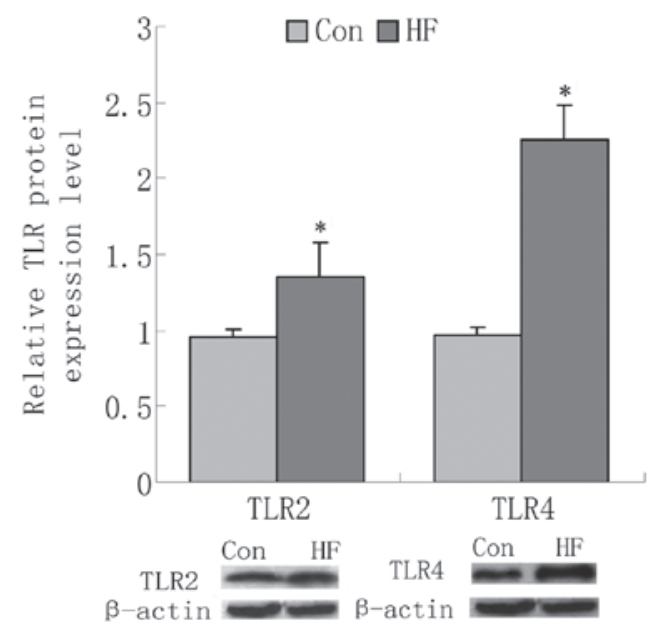

Figure 4. Comparison of TLR protein levels in the skeletal muscle of the Con group and the HF group as determined by western blot analysis. "P $<0.05$, compared with the Con group. HF, high-fat diet group; TLR, Toll-like receptor; Con, control.

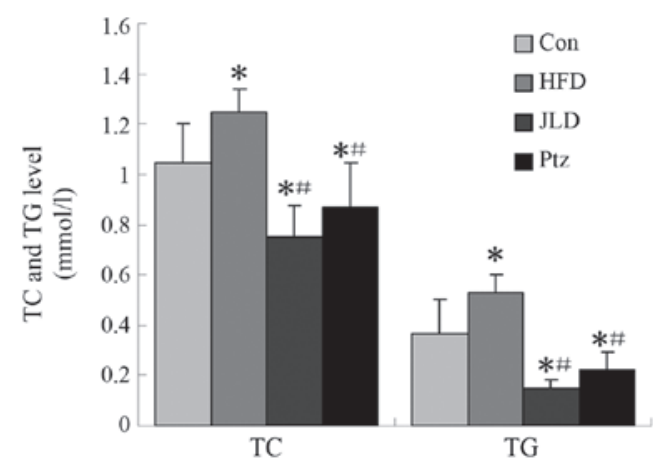

Figure 5. Comparison of TC and TG among different groups as determined by the 7300-110 apparatus. ${ }^{*} \mathrm{P}<0.05$, compared with Con group; ${ }^{~} \mathrm{P}<0.05$, compared with HFD group. Con, control group; HFD, control high-fat diet group; JLD, HFD+Jin Li Da treatment group; Ptz, HFD+pioglitazone treatment group; TC, cholesterol; TG, triglyceride.

and Fig. 1. The gene expression levels of TLR2 and TLR4 were significantly higher in the HTG and HTC groups compared with the Con group $(\mathrm{P}<0.05)$. The gene expression levels of TLR2 and TLR4 in the MHL group were also increased compared with that in the Con group (TLR2: $1.34 \pm 0.42$ vs. $0.98 \pm 0.32$, $\mathrm{P}<0.05$; TLR4: $1.63 \pm 0.41$ vs. $1.01 \pm 0.39, \mathrm{P}<0.05$ ).

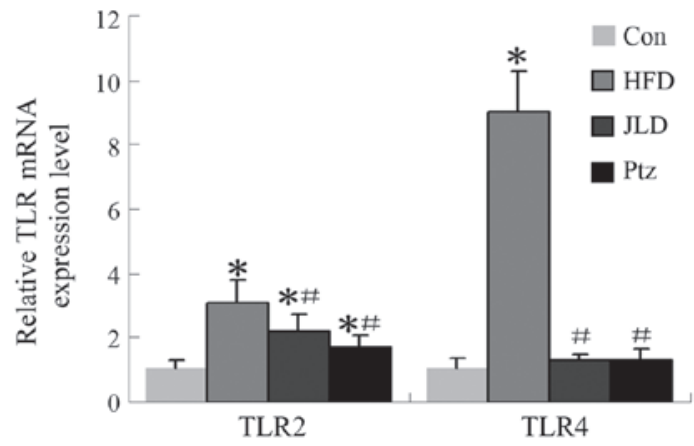

Figure 6. Comparison of TLR2 and TLR4 gene expression levels among the different treatment groups as determined by reverse transcription-quantitative polymerase chain reaction. ${ }^{*} \mathrm{P}<0.05$, compared with the Con group; ${ }^{*} \mathrm{P}<0.05$, compared with the HFD group. Con, control group; HFD, control high-fat diet group; JLD, HFD+Jin Li Da treatment group; Ptz, HFD+pioglitazone treatment group; TLR, Toll-like receptor.

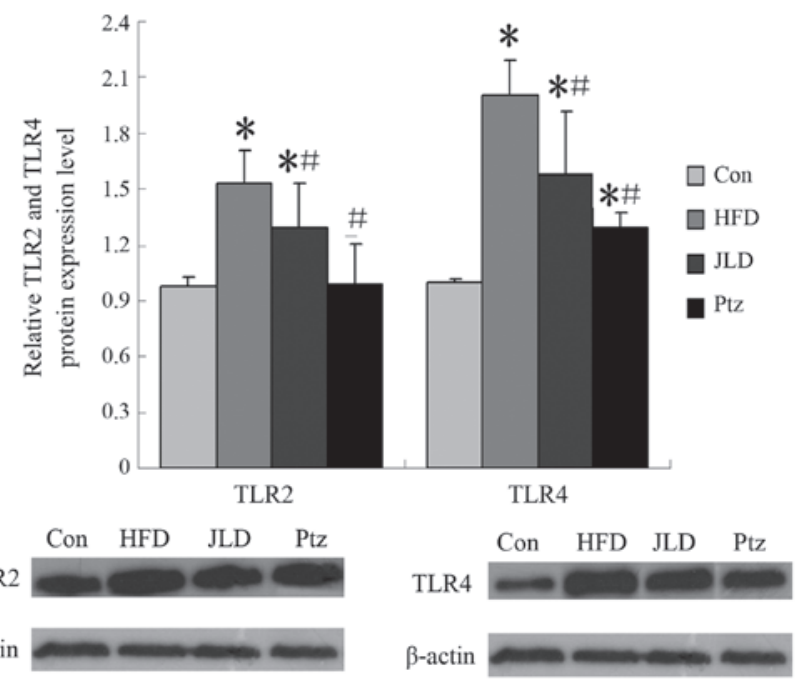

Figure 7. Comparison of TLR2 and TLR4 protein levels among different groups as determined by western blot analysis. ${ }^{*} \mathrm{P}<0.05$, compared with the Con group; ${ }^{\text {P }}<<0.05$, compared with the HFD group. Con, control group; HFD, control high-fat diet group; JLD, HFD+Jin Li Da treatment group; Ptz, HFD+pioglitazone treatment group; TLR, Toll-like receptor.

TLR2 and TLR4 expression in the skeletal muscle of the rat model. To further demonstrate the association between TLR2 and TLR4 with hyperlipidemia, a high-fat diet rat model was used. After 6 weeks on the high-fat diet, the TG and TC levels of the HF group were significantly higher compared with the $\mathrm{NC}$ group (Fig. 2). TLR2 and TLR4 gene expression in the skeletal muscle was detected using RT-qPCR. It was identified that the mRNA levels of two TLRs were upregulated in the HF group compared with the $\mathrm{NC}$ group, as were the protein levels (Figs. 3 and 4). To confirm the reliability of the results, lipid levels in the HF group were decreased through intragastric administration of JLD and Ptz. At the end of the 8-week drug intervention, TC, TG and TLR gene and protein levels in the skeletal muscle were analyzed again. From Fig. 5, it can be observed that levels of TC in the JLD and Ptz groups were decreased compared with the HFD group and NC group. TG levels were altered in the same manner. TLR gene and protein levels in skeletal muscle are shown in Figs. 6 and 7. TLR2 gene 
expression was significantly decreased in the JLD and Ptz groups compared with the HFD group $(\mathrm{P}<0.05)$, remaining higher than that of the $\mathrm{NC}$ group $(\mathrm{P}<0.05)$. The TLR2 protein level in the JLD group was significantly decreased and remained higher than the $\mathrm{NC}$ group $(\mathrm{P}<0.05)$. For the $\mathrm{Ptz}$ group, no significant differences were identified compared with the NC group ( $\mathrm{P}>0.05)$. TLR4 gene levels were significantly decreased in the JLD and Ptz groups compared with those in the HFD group and no significant differences were identified compared with the NC group. The protein levels in skeletal muscle also declined in the drug intervention groups, but remained higher than the $\mathrm{NC}$ group $(\mathrm{P}<0.05)$.

\section{Discussion}

The World Health Organization reports that obesity is one of today's most obvious public-health problems due to its high prevalence and its association with a wide range of chronic diseases, such as insulin resistance, type 2 diabetes, atherosclerosis, hypertension, immune-mediated disorders, certain types of cancer and NAFLD $(19,20)$. Chronic low level inflammation and hyperlipidemia, have been established to be key factors of obesity. TLRs mediate infection-induced inflammation by recognizing invading pathogens and activating downstream signaling pathways that lead to the expression of diverse arrays of pro-inflammatory marker gene products $(21,22)$. Recent evidence suggests that fatty acids are able to modulate TLR4 activation and that mice deficient in TLR2 were protected from high-fat diet-induced adiposity $(23,24)$.

In the present study, 250 patients were selected from the Medical Examination Center at Hebei General Hospital, including 84 patients with high levels of TC and normal levels of TG, 43 patients with high levels of TG and normal levels of TC, 55 patients with high TC and TG levels and 68 healthy controls with normal biochemical indicators. The present data revealed that TLR2 and TLR4 gene expression levels were significantly higher in the hyperlipidemia groups compared with the Con group. This suggests an association between the increase in TLR levels and and an increase in lipid levels.

In order to confirm the reliability of the results, a high-fat diet induced hyperlipidemia rat model was used, then the rats were protected from the high-fat diet-induced TC and TG increase through intragastric administration of JLD and Ptz. In our previous study, it was found that JLD had a significant protective effect against high-fat diet-induced lipid increase $(25,26)$. In addition, Ptz has been established to decrease hyperlipidemia and has been widely used in the clinical treatment of diabetes and combined hyperlipidemia $(2,27)$.

In the present study, it was identified that a 6-week high-fat diet is able to induce significant TC and TG increases, and JLD and Ptz were shown to significantly protect against these effects (Figs. 2 and 5). Although rats in the JLD and Ptz groups were fed a high-fat diet, their TC and TG levels were lower compared with the HFD group, and even lower than the Con group. However, it was also observed that TLR2 and TLR4 gene and protein expression levels in skeletal muscle were increased following a 6-weeks high-fat diet regimen, and their levels were decreased following a decrease in lipid levels.
Previous studies have established contribution of the TLR family to innate immunity and recent research has found that a high-fat meal induces low-grade endotoxemia, which is associated with obesity $(8,28,29)$. A further study reported that gut microbiota is a key modulator of insulin resistance (30), which together suggests a complex correlation between a high-fat diet, obesity, gut microbiota and insulin resistance. In the present study, the data revealed that TLR2 and TLR4 were increased in the hyperlipidemia groups and their gene and protein levels in skeletal muscle were consistent with the lipid level. This suggests that TLR2 and TLR4 were associated with the outcome of development of hyperlipidemia, however the mechanism for this requires further investigation.

In conclusion, for the first time, to the best of our knowledge the association between TLR and hyperlipidemia was demonstrated, and it was found that the levels of TLR 2 and TLR4 were correlated with the lipid level. The results suggest that TLRs are important in hyperlipidemia and may provide a deeper understanding of the mechanisms underlying hyperlipidemia for future studies.

\section{Acknowledgements}

This study was supported by grants from the National Natural Science Foundations of China (grant nos. 30971391 and 81170742) and the Hebei Natural Science Foundation of China (grant nos. C2010001638 and C2011307008).

\section{References}

1. López-Sánchez I, Sanz-Garcia M and Lazo PA: Plk3 interacts with and specifically phosphorylates VRK1 in Ser342, a downstream target in a pathway that induces Golgi fragmentation. Mol Cell Biol 29: 1189-1201, 2009.

2. Niranjan G, Mohanavalli V, Srinivasan AR and Ramesh R: Serum lipid peroxides and magnesium levels following three months of treatment with pioglitazone in patients with type-2 diabetes mellitus. Diabetes Metab Syndr 7: 35-37, 2013.

3. Ogden CL, Carroll MD, Curtin LR, Lamb MM and Flegal KM: Prevalence of high body mass index in US children and adolescents, 2007-2008. JAMA 303: 242-249, 2010.

4. Holl MG, Jaser SS, Womack JA, Jefferson VL and Grey M: Metabolic risk and health behaviors in minority youth at risk for type 2 diabetes. Diabetes Care 34: 193-197, 2011.

5. Sambataro M, Perseghin G, Lattuada G, Beltramello G, Luzi L and Pacini G: Lipid accumulation in overweight type 2 diabetic subjects: Relationships with insulin sensitivity and adipokines. Acta Diabetol 50: 301-307, 2013.

6. Bozzetto L, De Natale C, Di Capua L, Della Corte G, Patti L, Maione S, Riccardi G, Rivellese AA and Annuzzi G: The association of hs-CRP with fasting and postprandial plasma lipids in patients with type 2 diabetes is disrupted by dietary monounsaturated fatty acids. Acta Diabetol 50: 273-276, 2013.

7. Oda E: High-sensitivity C-reactive protein and white blood cell count equally predict development of the metabolic syndrome in a Japanese health screening population. Acta Diabetol 50: 633-638, 2013

8. Tilich M and Arora RR: Modulation of toll-like receptors by insulin. Am J Ther 18: e130-e137, 2011.

9. Lee JY, Ye J, Gao Z, Youn HS, Lee WH, Zhao L, Sizemore N and Hwang DH: Reciprocal modulation of Toll-like receptor-4 signaling pathways involving MyD88 and phosphatidylinositol 3-kinase/AKT by saturated and polyunsaturated fatty acids. J Biol Chem 278: 37041-37051, 2003.

10. Lee JY, Plakidas A, Lee WH, Heikkinen A, Chanmugam P, Bray G and Hwang DH: Differential modulation of toll-like receptors by fatty acids: Preferential inhibition by n-3 polyunsaturated fatty acids. J Lipid Res 44: 479-486, 2003. 
11. Lee JY, Zhao L, Youn HS, Weatherill AR, Tapping R, Feng L, Lee WH, Fitzgerald KA and Hwang DH: Saturated fatty acid activates but polyunsaturated fatty acid inhibits toll-like receptor 2 dimerized with toll-like receptor 6 or 1. J Biol Chem 279: 16971-16979, 2004.

12. Devaraj S, Dasu MR, Rockwood J, Winter W, Griffen SC and Jialal I: Increased toll-like receptor (TLR) 2 and TLR4 expression in monocytes from patients with type 1 diabetes: Further evidence of a proinflammatory state. J Clin Endocrinol Metab 93: 578-583, 2008.

13. Bian H, Yan H, Zeng M, Rao S, Yao X, Zhou J, Jia W and Gao X: Increased liver fat content and unfavorable glucose profiles in subjects without diabetes. Diabetes Technol Ther 13: 149-155, 2011

14. Devaraj S, Glaser N, Griffen S, Wang-Polagruto J, Miguelino E and Jialal I: Increased monocytic activity and biomarkers of inflammation in patients with type 1 diabetes. Diabetes 55: 774-779, 2006

15. Devaraj S, Cheung AT, Jialal I, Griffen SC, Nguyen D, Glaser N and Aoki T: Evidence of increased inflammation and microcirculatory abnormalities in patients with type 1 diabetes and their role in microvascular complications. Diabetes 56: 2790-2796, 2007.

16. Dasu MR, Devaraj S, Park S and Jialal I: Increased toll-like receptor (TLR) activation and TLR ligands in recently diagnosed type 2 diabetic subjects. Diabetes Care 33: 861-868, 2010.

17. Gan KX, Wang C, Chen JH, Zhu CJ and Song GY: Mitofusin-2 ameliorates high-fat diet-induced insulin resistance in liver of rats. World J Gastroenterol 19: 1572-1581, 2013.

18. Roth CL, Elfers CT, Figlewicz DP, Melhorn SJ, Morton GJ, Hoofnagle A, Yeh MM, Nelson JE and Kowdley KV: Vitamin D deficiency in obese rats exacerbates nonalcoholic fatty liver disease and increases hepatic resistin and toll-like receptor activation. Hepatology 55: 1103-1111, 2012.

19. Obesity: Preventing and managing the global epidemic. Report of a WHO consultation. World Health Organ Tech Rep Ser 894 i-xii, 1-253, 2000.

20. Choukem SP and Gautier JF: How to measure hepatic insulin resistance? Diabetes Metab 34 (6 Pt 2): 664-673, 2008.
21. Uematsu S and Akira S: Toll-like receptors and innate immunity. J Mol Med (Berl) 84: 712-725, 2006.

22. Kawai T and Akira S: Signaling to NF-kappaB by toll-like receptors. Trends Mol Med 13: 460-469, 2007.

23. Wong SW, Kwon MJ, Choi AM, Kim HP, Nakahira K and Hwang DH: Fatty acids modulate toll-like receptor 4 activation through regulation of receptor dimerization and recruitment into lipid rafts in a reactive oxygen species-dependent manner. J Biol Chem 284: 27384-27392, 2009.

24. Davis JE, Braucher DR, Walker-Daniels J and Spurlock ME: Absence of Tlr2 protects against high-fat diet-induced inflammation and results in greater insulin-stimulated glucose transport in cultured adipocytes. J Nutr Biochem 22: 136-141, 2011.

25. Zang SS, Song A, Liu YX, Wang C, Song GY, Li XL, Zhu YJ, Yu X, Li L, Liu CX, et al: Chinese medicine Jinlida (JLD) ameliorates high-fat-diet induced insulin resistance in rats by reducing lipid accumulation in skeletal muscle. Int J Clin Exp Med 8: 4620-4634, 2015.

26. Liu Y, Song A, Zang S, Wang C, Song G, Li X, Zhu Y, Yu X, Li L, Wang Y and Duan L: Jinlida reduces insulin resistance and ameliorates liver oxidative stress in high-fat fed rats. J Ethnopharmacol 162: 244-252, 2015.

27. Kurisu S, Iwasaki T, Ishibashi K, Mitsuba N, Dohi Y, Nishioka K and Kihara Y: Effects of low-dose pioglitazone on glucose control, lipid profiles, renin-angiotensin-aldosterone system and natriuretic peptides in diabetic patients with coronary artery disease. J Renin Angiotensin Aldosterone Syst 14: 51-55, 2013.

28. Sturton G, Persson C and Barnes PJ: Small airways: An important but neglected target in the treatment of obstructive airway diseases. Trends Pharmacol Sci 29: 340-345, 2008.

29. Kucuk T and Deveci S: Can 'chromohysteroscopy' help target endometrial biopsy in postmenopausal bleeding? Eur J Gynaecol Oncol 29: 165-167, 2008.

30. Wang S and Fischer PM: Cyclin-dependent kinase 9: A key transcriptional regulator and potential drug target in oncology, virology and cardiology. Trends Pharmacol Sci 29: 302-313, 2008. 